\title{
Infección pulmonar por Mycobacterium avium complex en el huésped inmunocompetente
}

\author{
FERNANDO SALDÍAS P.*, FERNANDO TIRAPEGUI S.** y ORLANDO DÍAZ P.*
}

\section{Mycobacterium avium complex pulmonary disease in immunocompetent adult patients}

Nontuberculous mycobacteria (NTM) are increasingly recognized as important pulmonary pathogens. Mycobacterium avium intracellulare complex (MAC) causes most lung infections due to NTM. Although the organism was identified in the 1890s, its potential to cause human disease was only recognized 50 years later. Patients with preexisting lung disease or immunodeficiency are at greatest risk for developing MAC infection. The majority of MAC pulmonary cases, however, occur in immunocompetent elderly women in association with nodular infiltrates and bronchiectasis. More recently, pulmonary disease has also been described in immunocompetent patients after exposure to MAC-contaminated hot tubs. We describe two cases of MAC lung disease in immunocompetent adult patients without preexisting lung disease and we review clinical manifestations, diagnostic criteria and treatment of this entity.

Key words: Mycobacterium avium complex, nontuberculous mycobacterium, bronchiectasis, diagnosis, treatment, prognosis.

\section{Resumen}

Las micobacterias no tuberculosas (MNT) se reconocen cada vez más como importantes patógenos pulmonares. El complejo Mycobacterium avium-intracellulare (MAC) causa la mayoría de las infecciones pulmonares por MNT. Aunque el organismo fue identificado en la década de 1890, su potencial patogenicidad en seres humanos fue reconocida sólo cincuenta años después. Los pacientes con enfermedad pulmonar preexistente o inmunodeficiencia están en mayor riesgo de desarrollar infección por MAC. Sin embargo, la mayoría de los casos se producen en mujeres de edad avanzada inmunocompetentes en asociación con infiltrados nodulares y bronquiectasias. Recientemente, la enfermedad pulmonar también se ha descrito en pacientes inmunocompetentes expuestos a equipos de hidroterapia o jacuzzis contaminados con MAC. En relación a dos pacientes adultos inmunocompetentes con enfermedad pulmonar por MAC examinamos el cuadro clínico, los criterios diagnósticos y el tratamiento de esta entidad.

Palabras clave: Mycobacterium avium complex, micobacterias no tuberculosas, bronquiectasias, diagnóstico, tratamiento, pronóstico.

\section{Introducción}

Las micobacterias no tuberculosas fueron descritas poco después que Koch identificó el bacilo tuberculoso y fueron denominadas "micobacterias atípicas" para diferenciarlas del Mycobacterium tuberculosis ${ }^{1}$. Inicialmente fueron consideradas microorganismos comensales ampliamente distribuidos en el ambiente (suelo, agua dulce y salada, animales domésticos y salvajes, leche y alimentos) $)^{2}$. A partir de 1950 se reconocieron como patógenos potenciales especialmente en el huésped con déficit de inmunidad celular (infección por VIH, linfoma, leucemias, trasplantes, quimioterapia) ${ }^{3}$. La clasificación de las micobacterias diseñada por Timpe y Runyon ${ }^{4}$, de acuerdo a sus características de crecimiento y pigmentación, ha permitido identificarlas y estudiarlas (Tabla 1).

* Departamento de Enfermedades Respiratorias, Facultad de Medicina, Pontificia Universidad Católica de Chile, Santiago de Chile.

** Servicio de Medicina, Hospital de los Ángeles, VIII Región, Chile. 
Tabla 1. Clasificación de las micobacterias no tuberculosas según Timpe y Runyon ${ }^{4}$

\begin{tabular}{|cl|}
\hline Grupo I & $\begin{array}{l}\text { Fotocromógenos: producen pigmento } \\
\text { amarillo en presencia de la luz }\end{array}$ \\
Especies: $M$. kansassi, M. marinum \\
Grupo II & $\begin{array}{l}\text { Escotocromógenos: producen pigmento } \\
\text { amarillo a naranja en la oscuridad }\end{array}$ \\
& Especies: $M$. scrofulaceum, $M$. gordonae \\
Grupo III & $\begin{array}{l}\text { No fotocromógenos: no producen } \\
\text { pigmento } \\
\text { Especies: } M . \text { avium, } M \text {. intracellulare }\end{array}$ \\
Grupo IV & $\begin{array}{l}\text { Rápido crecimiento: crecen en menos de } \\
\text { una semana } \\
\text { Especies: } M . \text { fortuitum, } M \text {. chelonae, } \\
\text { M. abscessus }\end{array}$ \\
\end{tabular}

En diferentes áreas geográficas, el complejo Mycobacterium avium (MAC) es el principal agente causal de enfermedad pulmonar, especialmente en pacientes con daño pulmonar estructural o en el huésped inmunocomprometi$\mathrm{do}^{5}$. El Mycobacterium avium y Mycobacterium intracellulare se distinguían por su patogenicidad en aves de corral y conejos, respectivamente ${ }^{6}$. En la actualidad, la mayoría de los laboratorios de microbiología informan $M$. avium-intracellulare o MAC porque las especies son similares. Las manifestaciones clínicas, radiológicas y la respuesta a la terapia antimicrobiana de los pacientes infectados por ambas especies son prácticamente indistinguibles ${ }^{7}$. En relación a dos casos clínicos revisaremos la epidemiología, etiopatogenia, cuadro clínico, diagnóstico y tratamiento de esta entidad.

\section{Casos clínicos}

\section{Caso 1}

Hombre de 55 años, no fumador, sin comorbilidades, consulta por episodios recurrentes de malestar general, fatigabilidad, sensación febril, sudoración, dorsalgia y tos seca de una a dos semanas de evolución, con una periodicidad de una a dos veces al año desde hace cinco años. La radiografía de tórax demuestra opacidades confluentes en ambos lóbulos superiores y língula que desaparecen con el tratamiento antimicrobiano (amoxicilina-ácido clavulánico, claritromicina o levofloxacina) en el curso de dos semanas. Se solicitó tomografía computada de tórax que demostró opacidades en árbol en brote y nódulos centrolobulillares en ambos lóbulos superiores y língula (Figura 1), la evaluación funcional respiratoria y el estudio inmunológico fueron normales y en el estudio microbiológico de muestras respiratorias repetidas (esputo y lavado broncoalveolar) hubo desarrollo de complejo Mycobacterium avium intracellulare. La identificación de especie por secuenciación de la región del gen para RNA ribosomal 16S confirmó la infección por $M$. avium. En el seguimiento clínico no ha habido progresión de las lesiones pulmonares y debido a la benignidad del cuadro clínico no ha recibido tratamiento específico.

\section{Caso 2}

Mujer de 65 años, no fumadora, portadora de rinosinusitis crónica y bronquiectasias bilaterales diagnosticados hace quince años, refiere exacerbaciones infecciosas de las bronquiectasias 2-3 veces al año que requieren tratamiento antibiótico prolongado. La radiografía de tórax demuestra opacidades nodulares peribronquiales difusas de predominio en el lóbulo medio y língula asociado a engrosamiento de paredes bronquiales (Figura 2) y la tomografía computada de tórax demuestra bronquiectasias, nódulos centrolobulillares y opacidades en árbol en brote en el lóbulo superior derecho, lóbulo medio y língula que han progresado en forma significativa en los últimos diez años. El estudio inmunológico (inmunoglobulinas séricas, subpoblaciones linfocitarias, serología de $\mathrm{VIH}$, anticuerpos antinucleares, anti DNA, $\mathrm{C}_{3}-\mathrm{C}_{4}$ séricos, factor reumatoideo, ANCA y perfil ENA) y la evaluación funcional respiratoria (espirometría, $\mathrm{DL}_{\mathrm{CO}}$ ) fueron normales, las baciloscopias de esputo fueron negativas y en el cultivo seriado de muestras respiratorias (esputo y lavado broncoal-

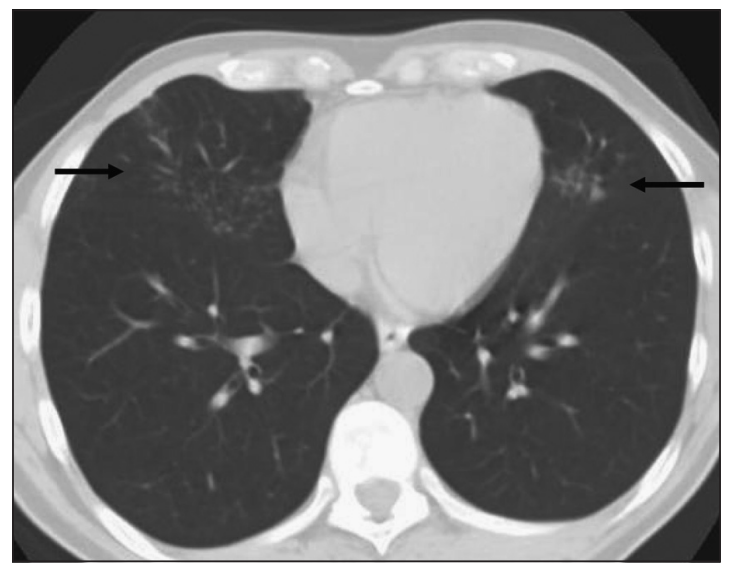

Figura 1. Tomografía computada de tórax de paciente inmunocompetente con infección por Mycobacterium avium-intracellulare. La imagen tomográfica muestra opacidades centrolobulillares con patrón de árbol en brote y bronquioloectasias en el lóbulo medio y língula (flechas). 


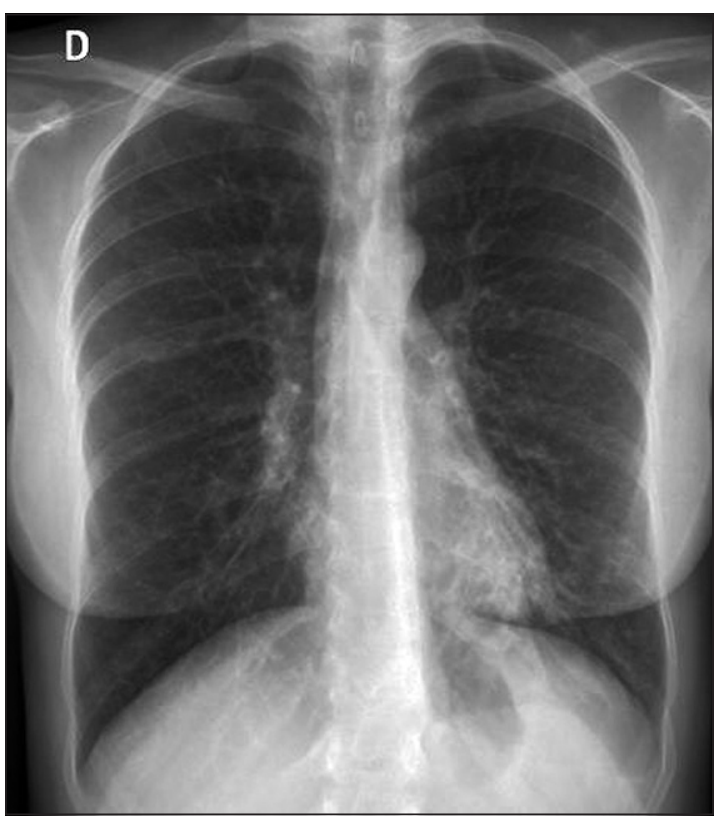

Figura 2. Mujer de 65 años inmunocompetente con infección por Mycobacterium avium-intracellulare. La radiografía de tórax evidencia opacidades areolares y engrosamiento peribronquial de predominio en el lóbulo superior derecho, lóbulo medio y língula.

veolar) hubo desarrollo de complejo $M$. avium intracellulare. Se recomendó iniciar tratamiento antibiótico específico.

\section{Discusión}

La presentación clínica de la infección por Mycobacterium avium intracellulare es variable . $^{8}$ El microorganismo puede ser aislado del esputo de individuos sanos (colonización), de pacientes con otras enfermedades pulmonares sin afectar su progresión, o puede causar progresión de la enfermedad pulmonar subyacente. El MAC puede causar enfermedad pulmonar progresiva que conduce a insuficiencia respiratoria e incluso a la muerte en pacientes previamente sanos, generalmente mujeres mayores sin antecedentes de enfermedad pulmonar o inmunodeficiencia ${ }^{8}$. La enfermedad diseminada se presenta en pacientes inmunocomprometidos, especialmente en pacientes con infección por virus de inmunodeficiencia humana ${ }^{9}$. En la infancia, excluyendo la fibrosis quística y SIDA, la enfermedad generalmente se presenta como linfadenopatía cervical.

Se ha comunicado un aumento de la prevalencia de infección pulmonar por $M$. avium en diferentes áreas geográficas ${ }^{8,10}$, lo cual puede ser explicado por el mayor desarrollo de las técnicas microbiológicas y de biología molecular que facilitan la identificación del microorganismo, el envejecimiento de la población y aumento de las comorbilidades que afectan el sistema inmune favoreciendo la infección pulmonar, y la vacunación con bacilo de Calmette-Guérin, lo cual le conferiría a la población cierta inmunidad ${ }^{11}$. Los pacientes con enfermedad pulmonar por MAC tendrían un perfil inmunológico diferente (menor producción de citoquinas protectoras: interferón gama, factor de necrosis tumoral alfa e interleuquina 12) comparado a la población sana con hipersensibilidad cutánea a antígenos de $\mathrm{MAC}^{12}$.

$\mathrm{Al}$ disminuir la prevalencia de la tuberculosis en la población mundial, con la introducción de una quimioterapia eficaz, se ha observado un aumento proporcional de los reportes de infección pulmonar por micobacterias no tuberculosas, especialmente por el complejo M. avium intracellulare, M. kansasii, M. malmoense, $M$. abscessus, y M. xenopi ${ }^{13}$. En 1980, un tercio de los aislamientos reportados a los Centros para el Control y Prevención de Enfermedades (CDC) eran micobacterias atípicas y $61 \%$ de ellos eran $\mathrm{MAC}^{14}$. En nuestro país se desconoce la real magnitud del problema; sin embargo, el 8,4\% de las muestras procesadas en el Laboratorio de Micobacteriología del Instituto de Salud Pública de Chile el año 2008 correspondieron a micobacterias ambientales, especialmente $M$. kansasii $(31,3 \%)$ y MAC $(18,8 \%)^{15}$.

La enfermedad pulmonar causada por MAC fue descrita por primera vez en pacientes hospitalizados con sospecha de tuberculosis, especialmente en adultos mayores con enfermedad pulmonar subyacente ${ }^{3,8}$. A diferencia de la tuberculosis, los pacientes con enfermedad pulmonar por MAC no son contagiosos, y no afectan a los miembros de la familia y otros contactos, incluidos los trabajadores de la salud.

La naturaleza insidiosa de esta enfermedad debe ser destacada, ya que los síntomas pueden estar presentes durante meses o años antes que se confirme el diagnóstico ${ }^{8,13}$ (Tabla 2). En las etapas iniciales de la enfermedad, los pacientes pueden ser asintomáticos y sólo se descubre por radiografías de tórax realizadas en controles de salud. La mayoría de los pacientes presentan tos crónica, con esputo purulento, habitualmente sin hemoptisis. Los síntomas constitucionales como malestar general, astenia, fatigabilidad, fiebre, sudoración nocturna, anorexia y pérdida de peso, no son comunes a menos que tengan enfermedad pulmonar extensa. Los pacientes con baciloscopia positiva de esputo son más propensos a tener fiebre y hemoptisis. La valoración de los síntomas 
Tabla 2. Manifestaciones clínicas de la enfermedad pulmonar por Mycobacterium avium complex ${ }^{13,17}$

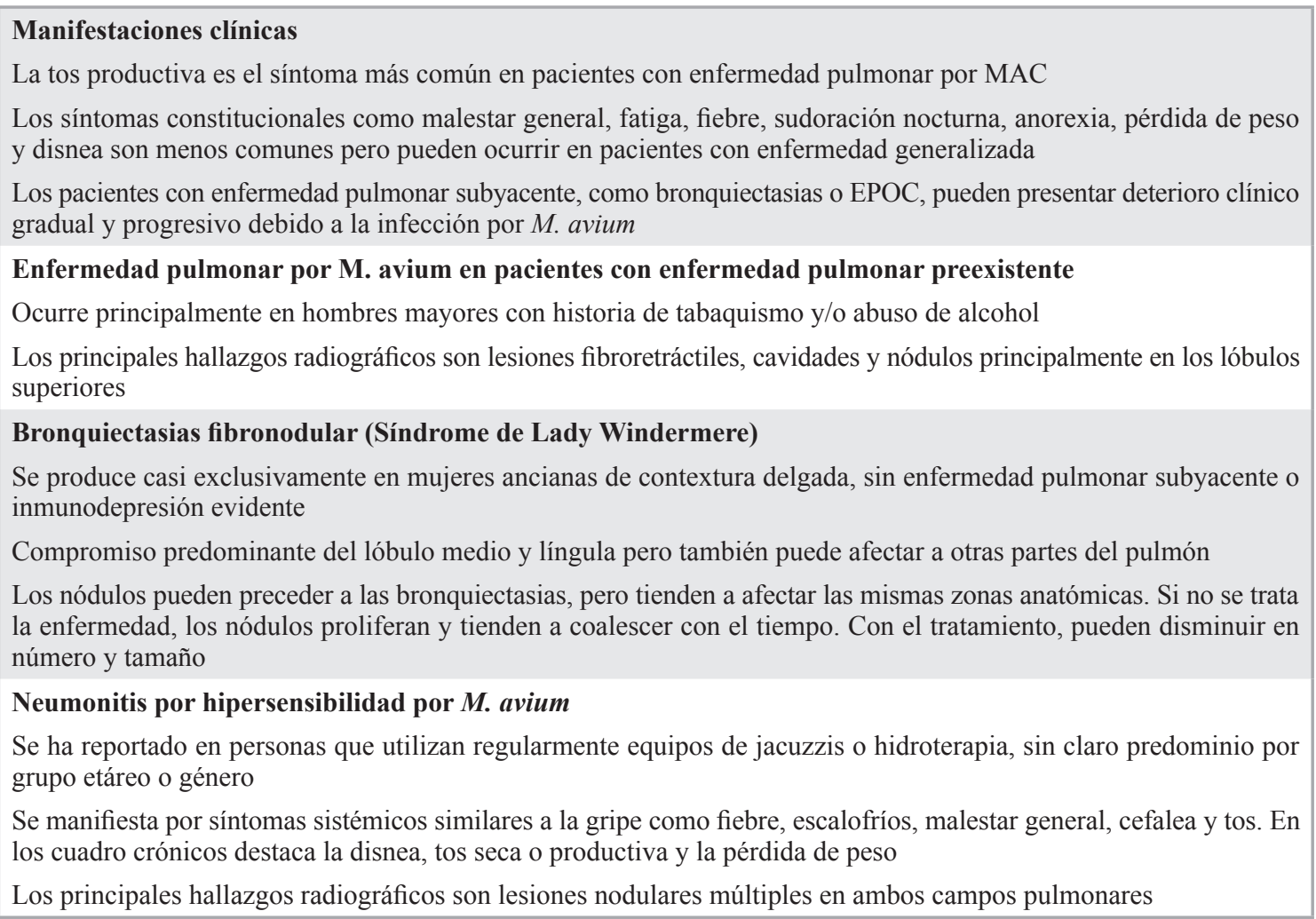

es complicada en los pacientes con enfermedad pulmonar preexistente. El tratamiento antimicrobiano específico debe mejorar los síntomas locales y generales de los enfermos.

En los reportes iniciales, la mayoría de los pacientes eran varones, tenían antecedentes de consumo de tabaco y alcohol y enfermedad pulmonar subyacente, y tenían enfermedad cavitaria de los lóbulos superiores ${ }^{16}$. En los informes más recientes, la mayoría de los pacientes son mujeres $\mathrm{o}$ ancianos con bronquiectasias ${ }^{17}$. En pacientes inmunocompetentes expuestos a equipos de hidroterapia o jacuzzis contaminados con MAC se ha descrito sensibilización antigénica, esta exposición produce una variedad de neumonitis por hipersensibilidad ${ }^{18}$. Los pacientes con fibrosis quística o portadores de un gen anormal de $\alpha_{1}$-antiproteasa parecen estar predispuestos a desarrollar esta condición ${ }^{17}$. No es clara su asociación a los antígenos del complejo mayor de histocompatibilidad.

Los pacientes con enfermedad pulmonar subyacente suelen tener enfermedad cavitaria en la radiografía de tórax ${ }^{8,13}$. Las cavidades serían más delgadas y con menos compromiso del parénquima circundante comparado con la tuberculosis.
En mujeres adultas sin enfermedad pulmonar subyacente, el compromiso de la vía aérea pequeña y bronquiectasias afectarían principalmente el lóbulo medio y la língula (síndrome de Lady Windermere). El derrame pleural no es común en la infección por MAC.

La tomografía computada es más sensible que la radiografía de tórax para la detección de las anormalidades estructurales asociadas a la enfermedad pulmonar por MAC ${ }^{13,17}$. La presencia de bronquiectasias y múltiples nódulos pulmonares pequeños son predictivos de enfermedad pulmonar por MAC, especialmente cuando son adyacentes entre sí. En una serie radiológica japonesa, la infección por MAC fue documentada mediante LBA y biopsia transbronquial en la mitad de los pacientes con nódulos centrolobulillares y bronquiectasias $^{19}$. Otros hallazgos radiográficos descritos en esta condición son atelectasias, consolidación del parénquima pulmonar, opacidades en "árbol en brote" y vidrio esmerilado con atrapamiento aéreo ${ }^{2,8,13}$. Los estudios de imágenes secuenciales han demostrado en algunos pacientes progresión de las lesiones de la vía aérea y bronquiectasias en el curso del tiempo ${ }^{17}$.

Los principales criterios diagnósticos de esta 
Tabla 3. Criterios diagnósticos de la enfermedad pulmonar por micobacterias no tuberculosas (MNT) ${ }^{22}$

La evaluación mínima de un paciente con sospecha de enfermedad pulmonar por micobacterias no tuberculosas debe incluir lo siguiente:

1) Radiografía de tórax o, en ausencia de cavitación, tomografía computarizada de tórax de alta resolución

2) Tres o más muestras respiratorias (esputo, aspirado bronquial, LBA, biopsia transbronquial) para análisis microbiológico de bacilos ácido alcohol resistentes

3) La exclusión de otras enfermedades, como la tuberculosis

Los criterios clínicos, radiológicos y microbiológicos son igualmente importantes y todos deben estar presentes para establecer el diagnóstico de enfermedad pulmonar por MNT

Los criterios diagnósticos se aplican a pacientes sintomáticos con opacidades radiográficas, nodulares o cavitarias, o que en la tomografía computarizada de tórax presentan bronquiectasias multifocales con múltiples nódulos pequeños

Estos criterios diagnósticos se aplican a pacientes con infección pulmonar por Mycobacterium avium complex, M. kansasii y M. abscessus. No se dispone de información suficiente que permita su aplicación universal a otras especies de MNT

Tabla 4. Recomendaciones de tratamiento de la enfermedad pulmonar por Mycobacterium avium complex ${ }^{22}$

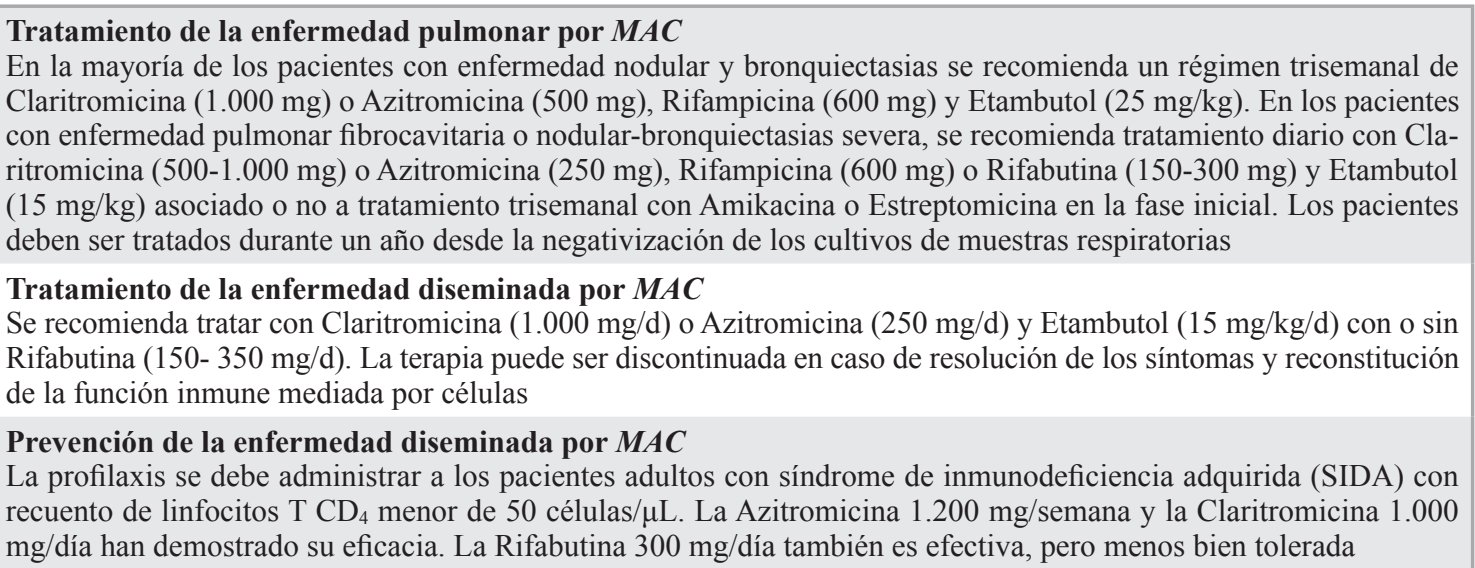

condición son un cuadro clínico-radiográfico compatible, la exclusión de otros diagnósticos y la demostración microbiológica de la micobacteria atípica en cultivos cuantitativos repetidos de muestras respiratorias ${ }^{13,17}$ (Tabla 3).

El tratamiento de la enfermedad pulmonar por el complejo $M$. avium intracellulare con medicamentos antituberculosos ha sido desalentador ${ }^{20-22}$. Las reacciones adversas de los medicamentos, los fracasos terapéuticos y recaídas frecuentes a menudo impiden una terapia exitosa $a^{21,22}$. La cirugía se ha recomendado en pacientes con enfermedad pulmonar localizada, especialmente en aquellos que no se logra erradicar el microorganismo del esputo con el tratamiento antimicrobiano ${ }^{23}$. Desde la introducción de los nuevos macrólidos, claritromicina y azitromicina, los resultados del tratamiento han mejorado.

Los regímenes de tratamiento para la enfermedad pulmonar por MAC no han sido claramente definidos (Tabla 4) y los resultados han sido desalentadores $^{20-22}$. El diagnóstico de esta condición no implica el inicio inmediato de tratamiento ya que algunos casos remiten espontáneamente. La decisión de tratar está basada en el análisis de cada caso particular, considerando el amplio espectro de presentaciones, desde el cuadro clínico indolente hasta el compromiso pulmonar progresivo con destrucción del parénquima pulmonar $^{20,22}$. Se ha observado daño pulmonar progresivo en sujetos asintomáticos, incluso con resultado de muerte, especialmente en pacientes con daño estructural previo, lesiones cavitadas y/o comorbilidad (EPOC, tabaquismo, alcoholismo). El tratamiento trisemanal con claritromicina o azitromicina, combinada con etambutol y rifampicina, ha demostrado ser eficaz ${ }^{21,22}$. Se recomienda mantener el tratamiento asociado durante doce meses desde que se negativizan las muestras de esputo, para evitar las recaídas precoces. 


\section{Bibliografía}

1.- FALKINHAM $\mathrm{J} O 3^{\text {rd }}$. Epidemiology of infection by nontuberculous mycobacteria. Clin Microbiol Rev 1996; 9: 177-215.

2.- FALKINHAM J O $3^{\text {rd }}$. Nontuberculous mycobacteria in the environment. Clin Chest Med 2002; 23: 529-51.

3.- PRIMM T P, LUCERO C A, FALKINHAM J O $3^{\text {rd }}$. Health impacts of environmental mycobacteria. Clin Microbiol Rev 2004; 17: 98-106.

4.- TIMPE A, RUNYON E H. The relationship of atypical acid-fast bacteria to human disease: a preliminary report. J Lab Clin Med 1954; 44: 202-9.

5.- O'BRIEN R J, GEITER L J, SNIDER D E Jr. The epidemiology of nontuberculous mycobacterial diseases in the United States. Results from a national survey. Am Rev Respir Dis 1987; 135: 1007-14.

6.- WOODS G L, WASHINGTON J A $2^{\text {nd }}$. Mycobacteria other than Mycobacterium tuberculosis: review of microbiologic and clinical aspects. Rev Infect Dis 1987; 9: 275-94.

7.- MAESAKI S, KOHNO S, KOGA H, MIYAZAKI Y, KAKU M. A clinical comparison between Mycobacterium avium and Mycobacterium intracellulare infections. Chest 1993; 104: 1408-11.

8.- PRINCE D S, PETERSON D D, STEINER R M, GOTTLIEB J E, SCOTT R, ISRAEL H L, et al. Infection with Mycobacterium avium complex in patients without predisposing conditions. N Engl J Med 1989; 321: 863-8.

9.- NIGHTINGALE S D, BYRD L T, SOUTHERN P M, JOCKUSCH J D, CAL S X, WYNNE B A. Incidence of Mycobacterium avium-intracellulare complex bacteremia in human immunodeficiency virus-positive patients. J Infect Dis 1992; 165: 1082-5.

10.- AHN C H, LOWELL J R, ONSTAD G D, SHUFORD E H, HURST G A. A demographic study of disease due to Mycobacterium kansasii or M intracellulare-avium in Texas. Chest 1979; 75: 120-5.

11.- TRNKA L, DANKOVÁ D, SVANDOVÁ E. Six years' experience with the discontinuation of BCG vaccination: 4. Protective effect of BCG vaccination against the Mycobacterium avium intracellulare complex. Tuber Lung Dis 1994; 75: 348-52.

12.- VANKAYALAPATI R, WIZEL B, SAMTEN B, GRIFFITH DE, SHAMS H, GALLAND M R, et al. Cytokine profiles in immunocompetent persons infected with Mycobacterium avium complex. J Infect Dis 2001; 183: 478-84.

13.- FIELD S K, COWIE R L. Lung disease due to the more common nontuberculous mycobacteria. Chest 2006; 129: 1653-72.

14.- GOOD R C, SNIDER D E Jr. Isolation of nontuberculous mycobacteria in the United States, 1980. J Infect Dis 1982; 146: 829-33.

15.- SCAPPATICCIO A, VELASCO M, LEIVA T, RODRÍ-
GUEZ J C. Frecuencia de micobacterias ambientales en Chile en el año 2008. Rev Chil Enf Respir 2011; 27: 214-22.

16.- CONTRERAS M A, CHEUNG O T, SANDERS D E, GOLDSTEIN R S. Pulmonary infection with nontuberculous mycobacteria. Am Rev Respir Dis 1988; 137 : 149-52.

17.- FIELD S K, FISHER D, COWIE R L. Mycobacterium avium complex pulmonary disease in patients without HIV infection. Chest 2004; 126: 566-81.

18.- EMBIL J, WARREN P, YAKRUS M, STARK R, CORNE S, FORREST D, et al. Pulmonary illness associated with exposure to Mycobacterium avium complex in hot tub water. Hypersensitivity pneumonitis or infection? Chest 1997; 111: 813-6.

19.- TANAKA E, AMITANI R, NIIMI A, SUZUKI K, MURAYAMA T, KUZE F. Yield of computed tomography and bronchoscopy for the diagnosis of Mycobacterium avium complex pulmonary disease. Am J Respir Crit Care Med 1997; 155: 2041-6.

20.- AMERICAN THORACIC SOCIETY. Diagnosis and treatment of disease caused by nontuberculous mycobacteria. Am J Respir Crit Care Med 1997; 156: S1-S25.

21.- GRIFFITH D E. Risk-benefit assessment of therapies for Mycobacterium avium complex infections. Drug Saf 1999; 21: 137-52.

22.- GRIFFITH D E, AKSAMIT T, BROWN-ELLIOTT B A, CATANZARO A, DALEY C, GORDIN F, et al, on behalf of the ATS Mycobacterial Diseases Subcommittee. An official ATS/IDSA statement: diagnosis, treatment, and prevention of nontuberculous mycobacterial diseases. Am J Respir Crit Care Med 2007; 175 : 367-416.

23.- SHIRIAISHI Y, NAKAJIMA Y, TAKASUNA K, HANAOKA T, KATSURAGI N, KONNO H. Surgery for Mycobacterium avium complex lung disease in the clarithromycin era. Eur J Cardiothorac Surg 2002; 21 : 314-8.

\section{Correspondencia a:}

Dr. Fernando Saldías Peñafiel

Departamento de Enfermedades Respiratorias

Pontificia Universidad Católica de Chile.

Teléfonos: 26331541 - 23543242

Fax: 26335255

Marcoleta 350 - 1er Piso

Santiago, Chile

Email: fsaldias@med.puc.cl 